\section{IMPACT OF A TELEMEDICINE LIFESTYLE-BASED REHABILITATION PROGRAM (HEAL) FOR GYNECOLOGIC CANCER PATIENTS}

N Gwacham*, N Mckenzie, J Pepe, S Ahmad, J Kendrick, R Holloway. AdvenHealth Cancer Institute, Gynecologic Oncology, Orlando, USA

\subsection{6/ijgc-2021-ESG0.571}

Introduction/Background* Cancer and its treatments can result in physical, psychological, and cognitive impairments. The Healthy Eating Active Lifestyle (HEAL) - GYN "rehabilitation" cancer program was developed to provide intensive group lifestyle training on exercise, nutrition, sleep, social integration, and stress management via a telemedicine platform. The aim of this study was to determine the impact of such an intervention on short-term quality of life for gynecologic cancer patients.

Methodology Gynecologic cancer patients underwent experiential instruction and personalized goal setting through an entirely virtual platform. A multidisciplinary team led by a gynecologic oncologist addressed diet, physical activity, strategies for sleep and stress management, sexual health, smoking cessation and alcohol intake. The intervention was aimed to address unmet psychosocial, emotional, physical, sexual, and spiritual needs common to cancer survivors. Self-administered questionnaires with Likert scales (1-5) were utilized in a preand post-fashion to assess improvements in physical activity, dietary and sleep habits, and a general medical symptom questionnaire (MSQ).

Result(s)* We report outcomes on the first 22 participants. The mean age was 58.8 years; 22 were Caucasian, and 7 patients were on maintenance therapy during their enrollment. There was a significant decrease in the average number of general symptoms reported by a comprehensive medical symptom questionnaire (MSQ) (36.39 vs 24.77, p<0.05). Trends towards improvement were demonstrated in eating patterns (4.59 vs $3.74 \mathrm{p}=0.06)$, perceived stress $(11.32$ vs 10.73 , $\mathrm{p}=0.28)$, levels of anxiety and depression (10.76 vs 7.68 , $\mathrm{p}=0.07)$ and weight management $(17.55$ vs $16.79, \mathrm{p}=0.23)$. Patients also reported feeling an increased sense of purpose and connection as a result of their enrollment in the program (35.57 vs $37.26, \mathrm{p}=0.07$ ). Effect sizes $(d)$ within the program were mild to moderate for all evaluated dimensions. $100 \%$ of participants would "highly recommend" the program and none complained of stress or altered mood associated with online instruction.

Conclusion* A telemedicine-based peri-habilitation program is feasible and well tolerated. Based on preliminary data, there are trends towards improvement in overall general medical symptoms, eating patterns, perceived stress, levels of anxiety and depression, and physical activity. These findings support continued investigation of a telemedicine-based healthy lifestyle peri-habilitative program.

\section{SURVEY OF MENOPAUSAL SYMPTOMS AFTER CANCER}

${ }^{1} \mathrm{H}$ Bartels ${ }^{*},{ }^{1} \mathrm{~F}$ Donohoe, ${ }^{2} \mathrm{~A}$ Roberts, ${ }^{2} \mathrm{~L}$ Comerford, ${ }^{3} \mathrm{Y}$ O'meara, ${ }^{1} \mathrm{D}$ Brennan. ${ }^{1}$ Ireland East Hospital Gynaecological Oncology Group, Mater Misericordiae University Hospital, Ireland, Gynae-oncology, Dublin, Ireland; ' ${ }^{2}$ Mater Misericordiae University Hospital, Gynaecological Oncology CNS - Survivorship SVUH/NMH, Dublin, Ireland; ' ${ }^{2}$ iving Well Cancer Programme, UCD Gynaecological Oncology Group (UCD-GOG) School of Medicine, Catherine McAuley Centre, Eccles Street, Dublin 7, Ireland, Dublin, Ireland

10.1136/ijgc-2021-ESGO.572
Introduction/Background* Women with a history of cancer often suffer with symptoms related to treatment long after their cancer diagnosis. The aim of this study is to explore the extent to which women experience menopausal symptoms following treatment, and the impact this has on their daily lives. Methodology Women with a history of cancer were invited to participate in an online survey in March 2020. Three separate surveys were completed which focused on menopausal symptoms, sleep disturbance and anxiety. Participants were recruited through patient support groups and via social media.

Result(s)* 418 women responded to the survey, with a median age of $49(23-72)$. Breast $(63 \%, n=265)$ and cervical $(17 \%$, $\mathrm{n}=71$ ) were the most prevalent cancers. The menopausal symptoms women found most troublesome and which they would most like to be rid of were hot flushes/night sweats (31\%, $n=96)$, disturbed sleep $(27 \%, n=85)$, and feeling tired or worn out $(19 \%, \mathrm{n}=56)$. Hot flushes severely interfered with women's quality of life, with $48 \%$ reporting "severe" sleep disturbance and 28\% $(n=98)$ felt their enjoyment of life was significantly impaired as a result. Sleep was severely affected by menopausal symptoms, with $80 \% \quad(n=324)$ of women reported waking up in the middle of the night or early morning and trouble falling asleep again. A significant number of women were using prescribed medication to aid sleep, with $30 \%(n=109)$ using medication more than once per week. Furthermore, $43 \%(n=150)$ women reported difficulty in staying awake while driving, eating or while socialising at least once a week. Questions relating to anxiety found $75 \%(n=273)$ were "unable to relax" during the past month and this was moderate to severe for $45 \%$ of women. Furthermore, a fear of dying was reported by $30 \%(n=186)$ of women.

Conclusion* This survey highlights the degree to which women suffer on a day to day basis following a cancer diagnosis and living with menopausal symptoms. Sleep disturbance and hot flushes were highlighted as particularly challenging. These data demonstrate the need to develop appropriate evidence based solutions and support services to manage menopausal symptoms after cancer treatment.

\section{SELF-MANAGEMENT AND ADHERENCE TO RECOMMENDED FOLLOW-UP AFTER GYNAECOLOGICAL CANCER: RESULTS FROM THE INTERNATIONAL INCHARGE STUDY}

${ }^{1} \mathrm{M}$ Skorstad, ${ }^{2,3} \mathrm{~B}$ De Rooij, ${ }^{4,5} \mathrm{MM}$ Jeppesen, ${ }^{6} \mathrm{SH}$ Bergholdt, ${ }^{7} \mathrm{PT}$ Jensen, ${ }^{2,3} \mathrm{~N}$ Ezendam, ${ }^{8,9} \mathrm{~K}$ Lindemann, ${ }^{10} \mathrm{~T}$ Bohlin, ${ }^{2,3,11} \mathrm{~L}$ Van de Poll-Franse, ${ }^{1,12} \mathrm{I}$ Vistad* ${ }^{*}$ 'Sorlandet Hospital HF Kristiansand, Dept. of Gynaecology and Obstetrics, Norway; ${ }^{2}$ Netherlands Comprehensive Cancer Organisation, Utrecht, Netherlands; ${ }^{3}$ CoRPS - Center of Research on Psychology in Somatic diseases, Department of Medical and Clinical psychology, Tilburg University, Tilburg, Netherlands; ${ }^{4}$ Lillebaelt Hospital, Dept. of Gynaecology and Obstetrics, Kolding, Denmark; ${ }^{5}$ Odense University Hospital, OPEN, Odense Patient data Explorative Network, Odense, Denmark; ${ }^{6}$ Odense University Hospital, Dept. of Gynaecology and Obstetrics, Odense, Denmark; ${ }^{7}$ Aarhus University Hospital and Aarhus University, Dept. of Gynaecology and Obstetrics, Aarhus, Denmark; ${ }^{8}$ Oslo University Hospital, Dept. of Gynaecologic oncology, Division of Cancer Medicine, Oslo, Norway; ${ }^{9}$ University of Oslo, Faculty of Medicine, Institute of Clinical Medicine, Oslo, Norway; ${ }^{10}$ Vestfold Hospital Trust, Dept. of Gynaecology and Obstetrics, Tonsberg, Norway; ${ }^{11}$ The Netherlands Cancer Institute, Division of Psychosocial Research and Epidemiology, Amsterdam, Netherlands; ${ }^{12}$ University of Bergen, Clinical Institute II, Medical department, Bergen, Norway

\subsection{6/ijgc-2021-ESG0.573}

Introduction/Background* The growing number of cancer survivors has led to increased focus on follow-up care. Resent 